\title{
Millimeter-Wave Diode-Grid Phase Shifters
}

\author{
WAYNE W. LAM, CHRISTINA F. JOU, HOWARD Z. CHEN, KJELL S. STOLT, \\ NEVILLE C. LUHMANN, JR., AND DAVID B. RUTLEDGE, MEMBER, IEEE
}

\begin{abstract}
Monolithic diode grids have been fabricated on $2 \mathrm{~cm}$ square gallium-arsenide wafers with 1600 Schottky-barrier varactor diodes. Shorted diodes are detected with a liquid-crystal technique, and the bad diodes are removed with an ultrasonic probe. A small-aperture reflectometer that uses wavefront division interference was developed to measure the reflection coefficient of the grids. A phase shift of $70^{\circ}$ with a $7 \mathrm{~dB}$ loss was obtained at $93 \mathrm{GHz}$ when the bias on the diode grid was changed from -3 $\mathrm{V}$ to $1 \mathrm{~V}$. A simple transmission-line grid model, together with the measured low-frequency parameters for the diodes, was shown to predict the measured performance over the entire capacitive bias range of the diodes, as well as over the complete reactive tuning range provided by a reflector behind the grid, and over a wide range of frequencies from 33 $\mathrm{GHz}$ to $141 \mathrm{GHz}$. This shows that the transmission-line model and the measured low-frequency diode parameters can be used to design an electronic beam-steering array and to predict its performance. An electronic beam-steering array made of a pair of grids using state-of-the-art diodes with $5 \Omega$ series resistances would have a loss of $1.4 \mathrm{~dB}$ at $90 \mathrm{GHz}$.
\end{abstract}

\section{INTRODUCTION}

NTERESTING millimeter-wave circuits become possible when solid-state devices are added to periodic structures. Lee and Fong made a pioneering study of the effect of embedding negative-resistance diodes in a corrugated grating [1]. Later, Alexopoulos et al. proposed using these active surfaces for scanning a beam electronically [2], and Chekroun et al. proposed Radant, a three-dimensional grid of diodes for steering a beam [3]. Rutledge and Schwarz demonstrated a multimode microbolometer array [4], and we proposed designs for a periodic grid loaded with diodes for electronic beam steering and frequency multiplication [5], [6]. In the beam-steering array (Fig. 1), the incident beam reflects off a pair of varactor diode grids, and the diode bias controls the phase of the reflected wave. The idea is to program a progressive phase shift across the aperture to steer the reflected beam. Diode grids are

Manuscript received July 16, 1987; revised December 11, 1987. This work was supported by the Army Research Office, by the U.S. Army Harry Diamond Laboratory, by the Jet Propulsion Laboratory, and by TRW under the University of California MICRO program.

W. W. Lam was with the Division of Engineering and Applied Science at the California Institute of Technology, Pasadena, CA 91125. He is now with the Military Electronics Division, TRW, Redondo Beach, CA 90270.

C. F. Jou and N. C. Luhmann, Jr., are with the Department of Electrical Engineering, University of California, Los Angeles, CA 90024.

H. Z. Chen and D. B. Rutledge are with the Division of Engineering and Applied Science, California Institute of Technology, Pasadena, CA 91125.

K. S. Stolt is with the Military Electronics Division, TRW, Redondo Beach, CA 90270.

IEEE Log Number 8819961.

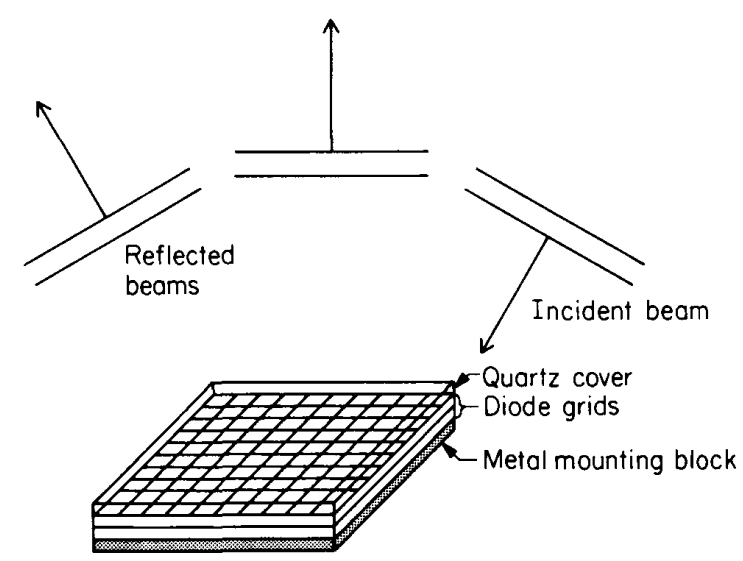

Fig. 1. Proposed millimeter-wave diode-grid electronic beam-steering array [5], [6].

attractive because they are suitable for monolithic integration. No transmission lines or waveguides are required, and this simplifies the design and fabrication. Since the power is distributed among many diodes over a large area, the power-handling capability can be quite high.

Fig. 2 shows the grid design and the equivalent transmission-line model, and a micrograph of one of the grids. The grid period is chosen to be about half the dielectric wavelength to avoid exciting substrate modes [5]. The vertical metal strips are inductive leads for the diodes, while the horizontal strips are for biasing the diodes by rows. The electric field is vertically polarized. In the circuit model (Fig. 2(b)), the grid is represented by an inductor in series with a diode, and the substrate is represented by a section of transmission line with a characteristic impedance equal to the wave impedance in the dielectric. The horizontal bias leads are neglected because they are perpendicular to the incident electric field. This design only allows the reflection phase to be varied from row to row, so that we would only be able to scan in the $E$ plane. To scan in both planes, we would need to bias diodes individually, or add additional grids with diodes connected in series rather than in parallel. The grid inductance is given by the quasi-static formula [5]

$$
L=\left(\frac{\mu_{0} a}{2 \pi}\right) \ln \left[\csc \left(\frac{\pi w}{2 a}\right)\right]
$$




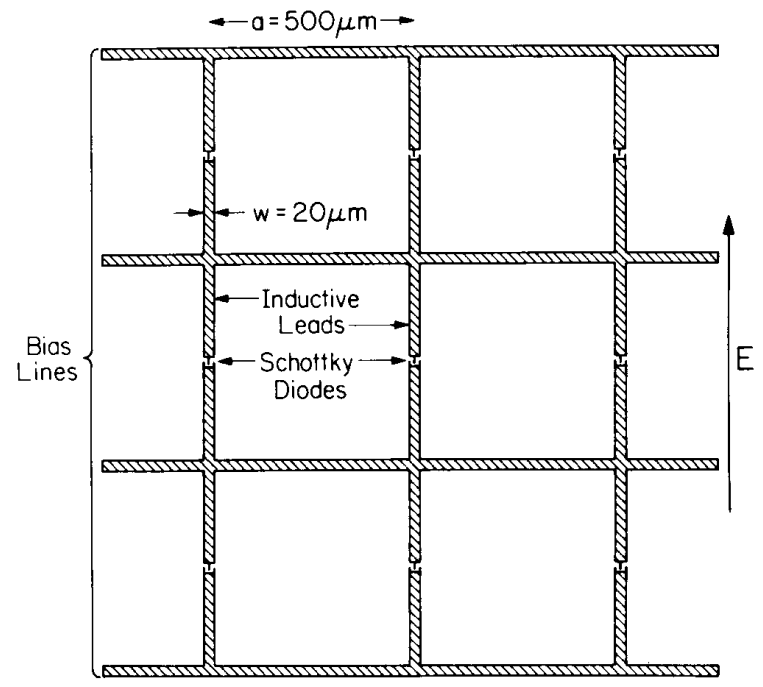

(a)

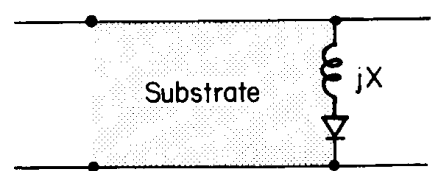

(b)

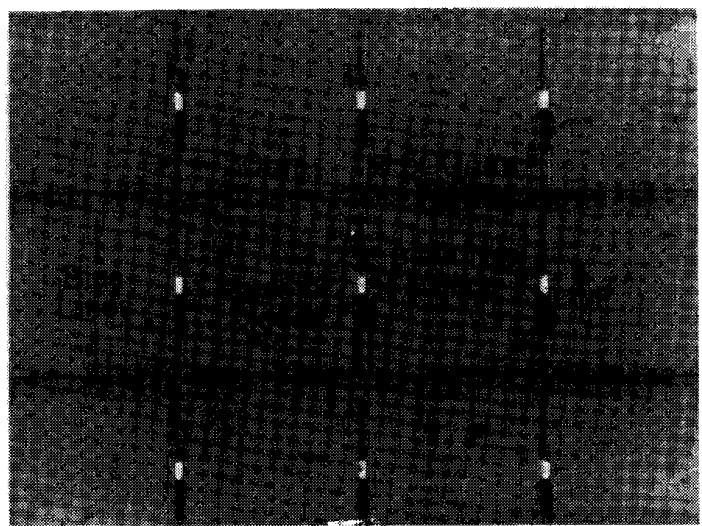

(c)

Fig. 2. (a) Diode-grid dimensions, (b) the equivalent transmission-line model, and (c) a micrograph of a section of a diode grid fabricated on a gallium-arsenide wafer.

where $a$ is the grid period and $w$ is the lead width. For our grid, with $a=500 \mu \mathrm{m}$ and $w=20 \mu \mathrm{m}$, the inductance is $277 \mathrm{pH}$, which gives a reactance of $157 \Omega$ at $90 \mathrm{GHz}$. This inductance is in series with the diode capacitance, and it allows the total reactance to be tuned over a range of both positive and negative reactances.

\section{FABRICATION}

Four grids were made. The wafer area ranged from 4 $\mathrm{cm}^{2}$ to $6 \mathrm{~cm}^{2}$, and the wafers were lapped to a thickness of $230 \pm 25 \mu \mathrm{m}$, which is a quarter of a dielectric wavelength at $90 \mathrm{GHz}$. The varactor diodes were fabricated with a self-aligning process similar to that developed by Zah et al.

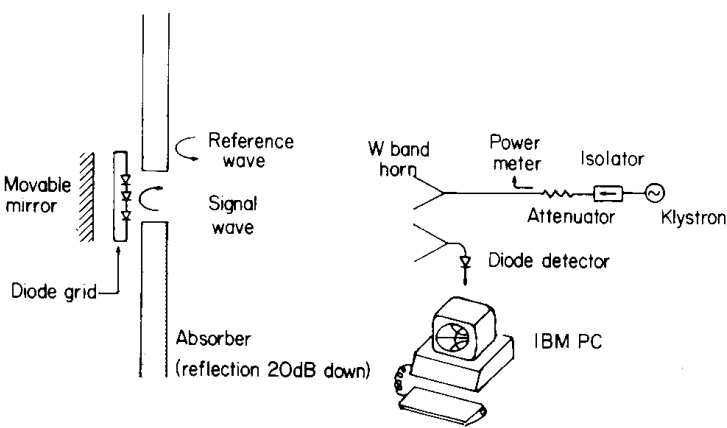

Fig. 3. Computer-controlled reflectometer for measuring reflection coefficients of small irregular samples at millimeter-wave frequencies.

[7]. Details are given in [6]. The epitaxial layers were grown on a semi-insulating gallium arsenide substrate by molecular beam epitaxy, and a truncated hyperabrupt $x^{-1}$ doping profile was used. The $x^{-1}$ doping profile causes the reactance to tune linearly with the applied bias voltage. The diode area was $18 \mu \mathrm{m}^{2}$. The best fabrication yield for individual diodes in an array was 98 percent. The remaining shorted diodes were identified with a liquid crystal detection technique, and these were removed with an ultrasonic probe. The measured diode series resistances ranged from $20 \Omega$ to $100 \Omega$. The zero-bias capacitance of the individual diodes at $1 \mathrm{MHz}$ ranged from $20 \mathrm{fF}$ to $40 \mathrm{fF}$, and the breakdown voltage of the diode grid ranged from $-1 \mathrm{~V}$ to $-3 \mathrm{~V}$. The low breakdown voltage is partly attributed to the fact that aluminum was evaporated in an oil diffusion-pumped vacuum system rather than grown in situ by molecular beam epitaxy [8].

\section{Reflection MEasurements}

Fig. 3 shows a computer-controlled small-aperture reflectometer we developed to measure the reflection coefficient of the diode grid. The idea is to use an absorbing foam screen (Eccosorb AN-72, manufactured by Emerson and Cuming) with a hole in the center to divide an incident wavefront into two parts. At millimeter-wave frequencies, this foam is not a perfect absorber, and it reflects about 1 percent of the incident power. The portion that reflects off the absorber is the reference, while the other part, which reflects off the sample, is the signal. The interference between these waves is measured as the sample is translated relative to the absorber. The phase and the amplitude of the reflection coefficient were calculated by a simple four-point method [9]. This method of measurement is attractive for small and irregular samples, because the absorber hole can be cut to the shape of the sample, and the supporting apparatus is shielded from the incident waves. In addition, there are no lenses to contribute stray reflections. The initial distance between the sample and the mirror is measured by a small hand-held microscope to an accuracy of $25 \mu \mathrm{m}$. The scanning mirror serves as a tuning parameter as well as a standard load for calibration. The mirror was a good reference for reflectance, but in the phase measurements there were offsets of about $10^{\circ}$, pre- 


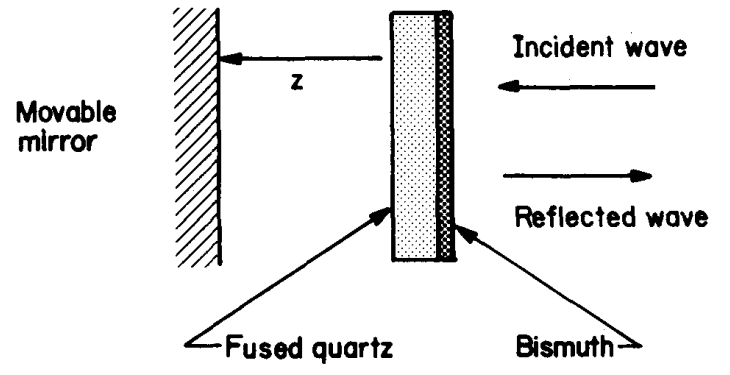

(a)

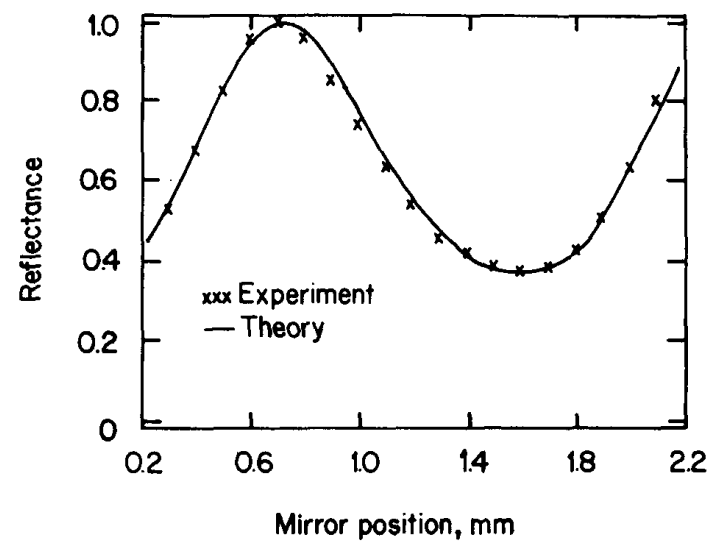

(b)

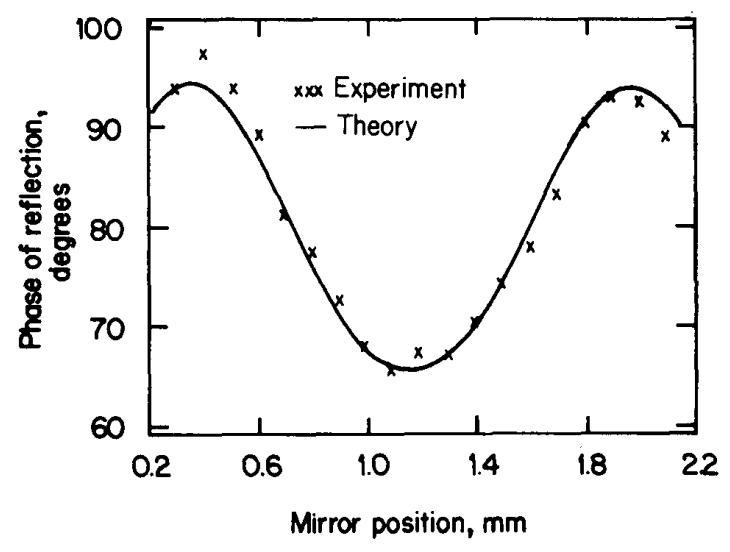

(c)

Fig 4. (a) Bismuth-on-quartz test structure for small-aperture reflectometer. (b) Reflectance and (c) phase as a function of mirror position at $93 \mathrm{GHz}$. The theory uses the transmission-line model and $\mathrm{dc}$ four-point probe measurements of the bismuth resistance.

sumably due to errors in measuring distances. For this reason, a phase offset was included as a free parameter to be determined by curve fitting. Effectively the tuning curves are used to calibrate the phase. The slab samples and the scanning mirror are aligned with a helium-neon laser, and are translated by stepper motors with a resolution of $1 \mu \mathrm{m}$ per step.

To test the reflectometer, the reflection coefficient of a $2.5 \mathrm{~cm}$ square fused-quartz plate coated with a $600 \AA$ thick bismuth film was measured and compared with transmission line theory (Fig. 4). The theoretical curves are plotted

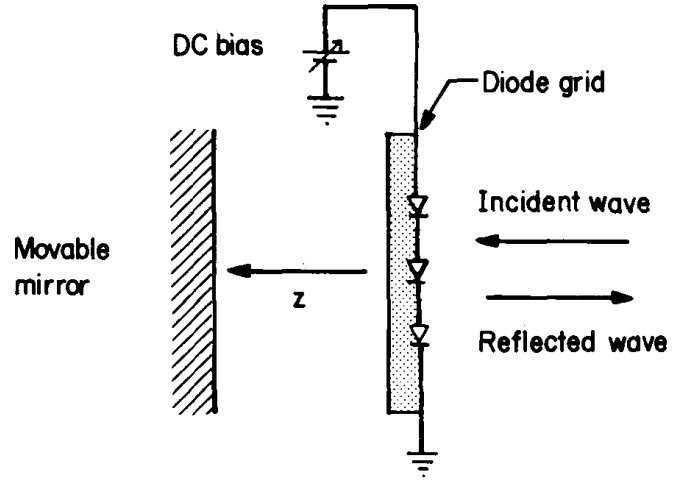

(a)

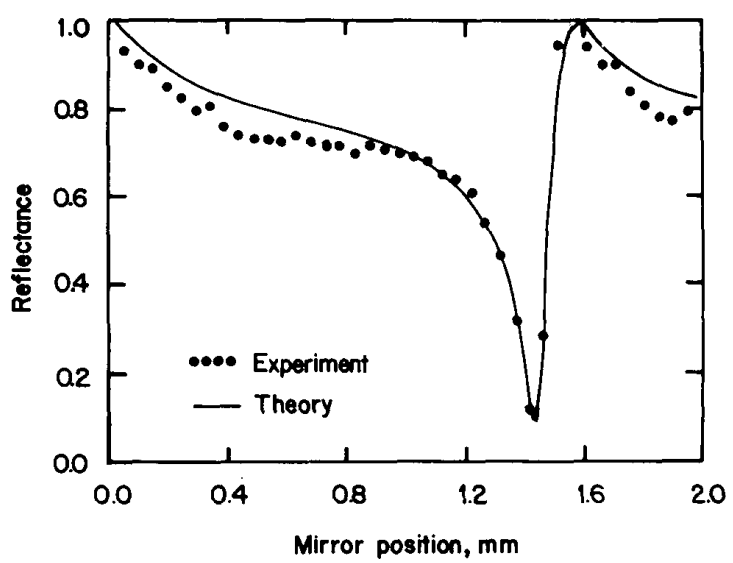

(b)

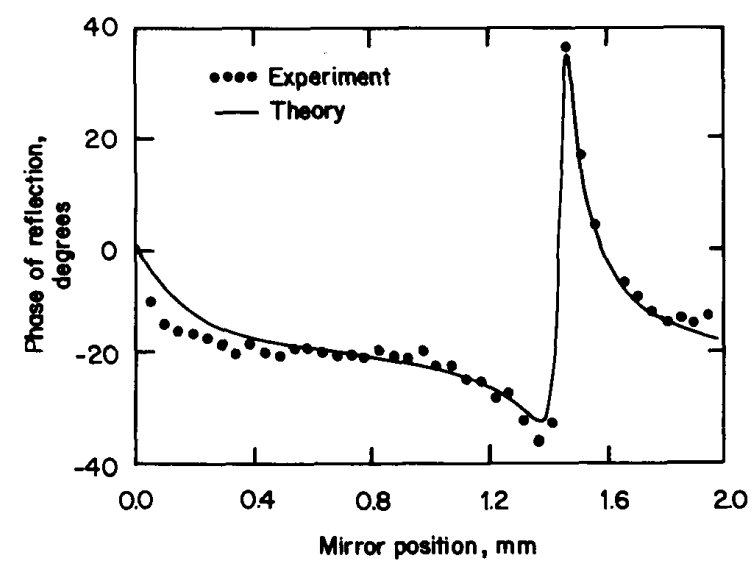

(c)

Fig. 5. (a) Testing the diode grid. (b) Reflectance and (c) phase as a function of mirror position at zero bias and $93 \mathrm{GHz}$. The theory is based on the transmission-line model and low-frequency measurements on the diodes.

using the dc four-point-probe measurement of the bismuth sheet resistance $(92.2 \Omega)$ and the measured plate thickness $(434 \pm 5 \mu \mathrm{m})$. The refractive index of fused quartz, 1.96, was taken from the work of Afsar and Button [10]. We also used the curve-fitting routine to work backward and predict the sheet resistance from the millimeter-wave experi- 


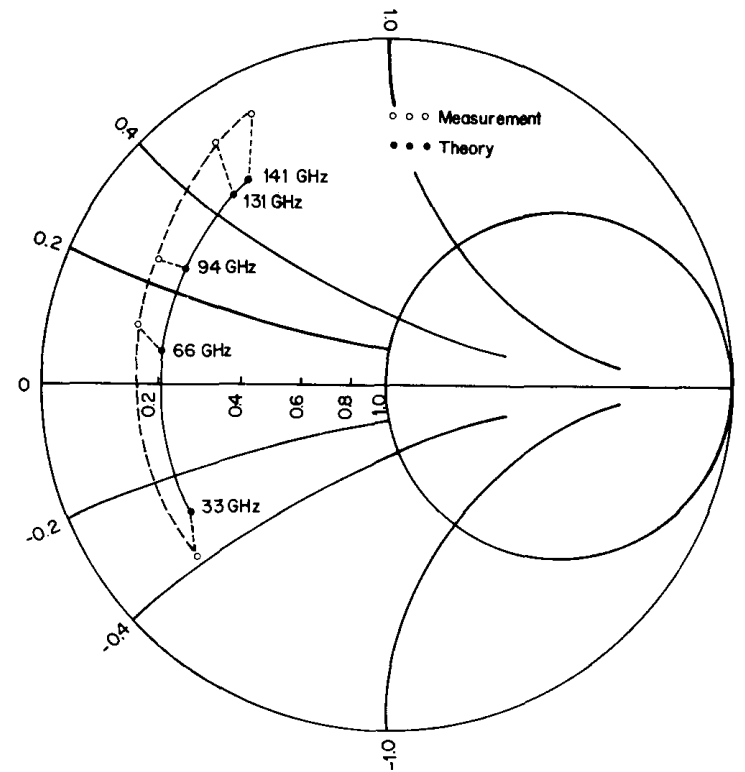

Fig. 6. Diode-grid impedance as a function of frequency, plotted on a Smith chart with a normalizing impedance of $377 \Omega$. The theoretical curve uses the measured average dc series resistance, $78 \Omega$, the measured average $1 \mathrm{MHz}$ capacitance, $30 \mathrm{fF}$, and the quasi-static inductance, $277 \mathrm{pH}$. The plot shows a characteristic clockwise spiral as the frequency increases.

mental curves. The RF result was $91.6 \Omega$, which compares well with the dc value of $92.2 \Omega$.

\section{Characterizing the Diode Grids}

These measurements gave us the confidence to use the reflectometer to characterize the diode grids. We measured the reflection coefficient of a grid as a function of mirror distance at $93 \mathrm{GHz}$, and the reflectance and the phase are plotted in Fig. 5. Also plotted are theoretical curves based on the transmission-line model of the grid, with the Afsar and Button values for the refractive index of gallium arsenide, $3.6[10]$. One complicating factor is that the thickness of the gallium-arsenide wafers was not uniform. The wafers were lapped by hand, and the thickness at different parts of the wafer varied by $\pm 25 \mu \mathrm{m}$. This thickness and the separation between the wafer and the mirror were left as free parameters, and determined by curve fitting. For the diode parameters we used $1 \mathrm{MHz}$ capacitance measurements on individual diodes in the grid (mean value, $30 \mathrm{fF}$ ) and dc series resistance measurements on individual diodes (mean value, $78 \Omega$; standard deviation, $19 \Omega$ ). In addition, we used the reflectance and phase curves in Fig. 5 and transmission line theory to de-embed the grid impedance from the wafer and the mirror. For this grid, the impedance at $93 \mathrm{GHz}$ is $58+j 94 \Omega$. The real part compares reasonably well with the dc series resistance measurements quoted above. We extended these measurements with several different oscillators to measure the grid impedance at frequencies from $33 \mathrm{GHz}$ to $140 \mathrm{GHz}$. The results are plotted on a Smith chart in Fig. 6.

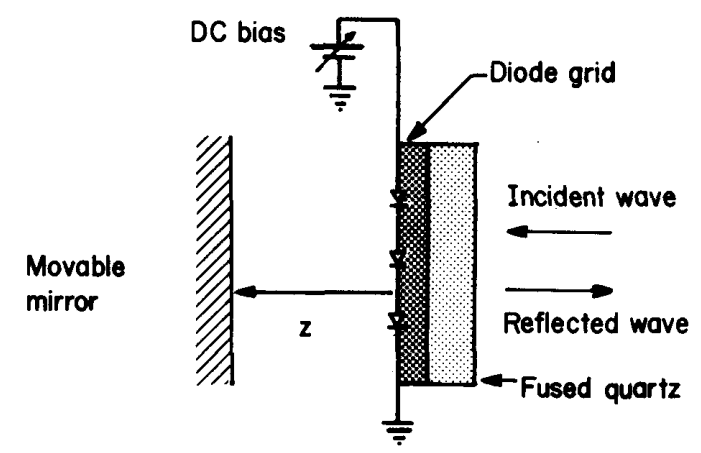

(a)

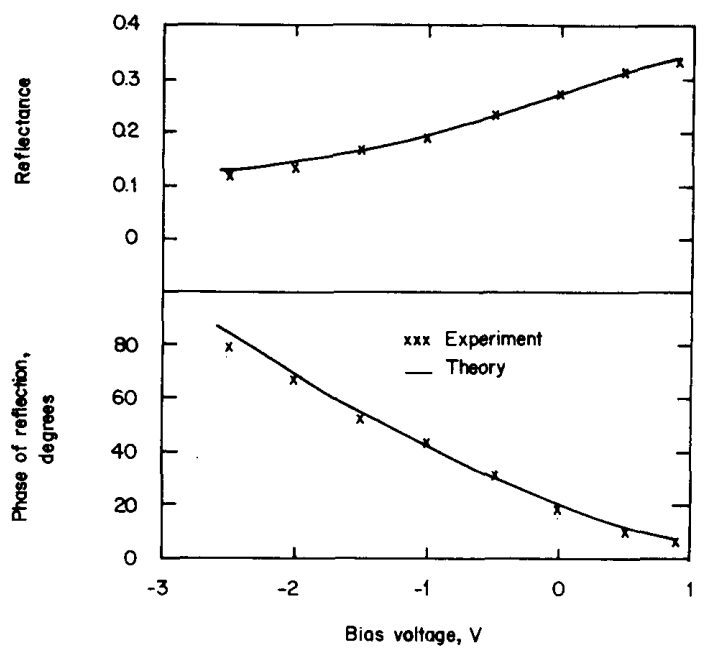

(b)

Fig. 7. (a) The phase shift measurement at $93 \mathrm{GHz}$. The back side of the gallium-arsenide wafer faces away from the mirror, and a quarterwave layer of fused quartz has been added. The mirror was placed 1.49 $\mathrm{mm}$ from the diode grid. (b) Phase shift and loss of a diode grid at 93 $\mathrm{GHz}$.

The phase shift performance was measured at $93 \mathrm{GHz}$ with a different diode grid with a lower dc series resistance, $26 \Omega$. Fig. 7 shows the configuration and a comparison between experiment and theory based on the measured low-frequency capacitance variation between $27 \mathrm{fF}$ at zero bias, and $20 \mathrm{fF}$ at $-3 \mathrm{~V}$. A $70^{\circ}$ phase shift with an average loss of $7 \mathrm{~dB}$ was measured. The low breakdown voltage limited the phase shift that could be obtained, and the high series resistance accounts for the loss. It should be noted that at this point in the measurements, three out of 35 rows of the diode grid were shorted out and could not be biased.

\section{Conclusions}

In these plots, theory and experiment agree well over the complete reactive tuning range provided by the mirror, over a wide band of frequencies from $33 \mathrm{GHz}$ to $141 \mathrm{GHz}$, and over the entire capacitive voltage bias range of the diodes. This shows that we can use the simple transmission line model and the measured low-frequency diode parame- 


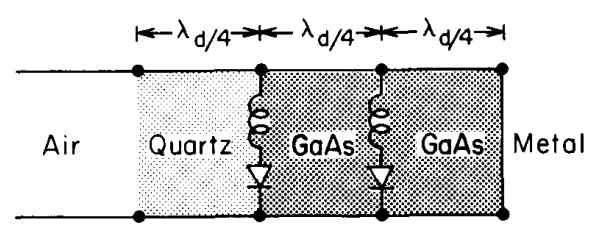

(a)

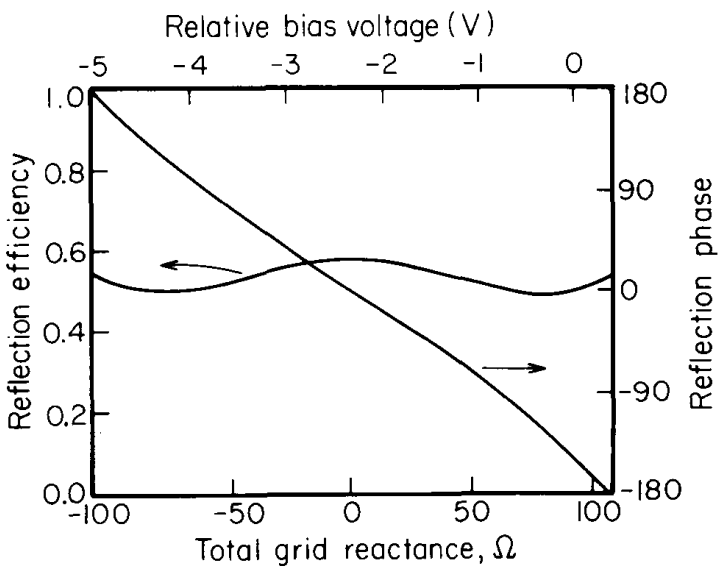

(b)

Fig. 8. (a) Beam-steering array with two grids [5], and (b) the simulated reflectance and phase shift for a diode series resistance of $10 \Omega$. The incident waves are TE polarized at an incident angle of $45^{\circ}$

ters to design a beam-steering array and to predict its performance. To build a beam-steering array, we need to be able to vary the phase by $360^{\circ}$. This is difficult to do with a single grid, but a design for two grids is shown in Fig. 8. There are two identical grids with a reflecting mirror on the back and a quartz matching layer on the front. It is an analog to an earlier phase shifter developed by Garver [11]. To make a $360^{\circ}$ phase shift, the total reactance on each grid must sweep from $+107 \Omega$ to $-107 \Omega$, where $107 \Omega$ is the wave impedance of the gallium arsenide substrate. In the simulation, the series resistance is assumed to be $10 \Omega$. We found that the average loss is $2.7 \mathrm{~dB}$. In addition, the simulations show that the loss in $\mathrm{dB}$ varies almost linearly with the series resistance. Since researchers have reported millimeter-wave monolithic diodes with series resistances as low as $5 \Omega$ [12], [13], it is now feasible to make an electronic beam-steering array at $90 \mathrm{GHz}$ with a loss of $1.4 \mathrm{~dB}$.

\section{ACKNOWLEDGMENT}

One of the authors (W. Lam) acknowledges the support of an AMOCO Foundation Fellowship and would like to thank Dr. Chung-en Zah at Bell Communication Research for showing him how to fabricate diodes. The authors are indebted to Prof. A. Yariv and Prof. M-A. Nicolet at Caltech for the use of their fabrication facilities, and to Prof. W. Bridges at Caltech for the loan of millimeter-wave

equipment. They would also like to thank Dr. T. Kuech at IBM for providing MOCVD wafers, Dr. T. Fong and Dr. John Berenz at TRW and Dr. J. Maserjian and L. Eng at JPL for providing MBE wafers, Dr. H. Yamasaki, Dr. H. Kanber, and B. Rush at Hughes, and Dr. F. So and A. Ghaffari at Caltech for providing proton implantation.

\section{REFERENCES}

[1] S. W. Lee and T. T. Fong, "Electromagnetic wave scattering from an active corrugated structure," J. Appl. Phys., vol. 33, pp. 388-396, Feb. 1972.

[2] N. G. Alexopoulos, P. L. E. Uslenghi, and G. A. Tadler, "Antenna beam scanning by active impedance loading," IEEE Trans. Antennas Propagat., vol. AP-22, pp. 722-723, Sept. 1974.

[3] C. Chekroun, D. Herrick, Y. Michel, R. Pauchard, and P. Vidal, "Radant: New method of electronic scanning," Microwave J., pp. 45-53, Feb. 1981

[4] D. B. Rutledge and S. E. Schwarz, "Planar multimode detector arrays for infrared and millimeter-wave applications," IEEE J. Quantum Electron., vol. QE-17, pp. 407-414, 1981

[5] W. W. Lam, C. F. Jou, N. C. Luhmann, Jr., and D. B. Rutledge, "Diode grids for electronic beam steering and frequency multiplication," Int. J. Infrared and Millimeter Waves, vol. 7, pp. 27-41, 1986. (Note: There is an error in the paragraph at the bottom of $p$. 35. Change $20 \Omega$ to $10 \Omega$.)

[6] W. W. Lam, “Millimeter-wave monolithic Schottky diode-grid phase shifters," Ph.D. thesis, California Institute of Technology, 1987.

[7] C. E. Zah, D. Kasilingam, J. S. Smith, D. B. Rutledge, T. C. Wang, and S. E. Schwarz, "Millimeter-wave monolithic Schottky-diode imaging arrays," Int. J. Infrared and Millimeter Waves, vol. 6, pp. 981-997, 1986.

[8] Y. Sato, M. Uchida, K. Shimada, M. Ida, and T. Imai, "GaAs Schottky barrier diode, ECL-1314," Rev. Elect. Commun. Lah., vol. 18, pp. 638-644, 1970.

[9] J. C. Wyant, "Interferometry for three-dimensional sensing," Test and Measurement World, pp. 66-71, Apr. 1986

[10] M. N. Afsar and K. J.Button, "Precise millimeter-wave measurements for complex refractive index, complex dielectric permittivity and loss tangent of $\mathrm{GaAs}, \mathrm{Si}, \mathrm{SiO}_{2}, \mathrm{Al}_{2} \mathrm{O}_{3}, \mathrm{BeO}, \mathrm{Macor}$, and glass," IEEE Trans. Microwave Theory Tech., vol. MTT-31, pp. 217-223, 1983.

[11] R. V. Garver, " $360^{\circ}$ varactor linear phase modulator," IEEE Trans. Microwave Theory Tech., vol. MTT-17, pp. 137-147, 1969.

[12] B. J. Clifton, G. D. Alley, R. A. Murphy, and I. H. Mroczkowski, "High performance quasi-optical GaAs monolithic mixer at 110 GHz," IEEE Trans. Electron Devices, vol. ED-28, pp. 155-157, 1981 .

[13] W. L. Bishop, K. L. McKinney, R. J. Mattauch, T. W. Crowe, and G. Green, "A novel whiskerless Schottky diode for millimeter and submillimeter wave application," in 1987 IEEE MTT-S Int. Symp. Dig. (Las Vegas, NV), June 1987, pp. 607-610.

\section{斗}

Wayne W. Lam, photograph and biography not available at the time of publication.

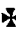

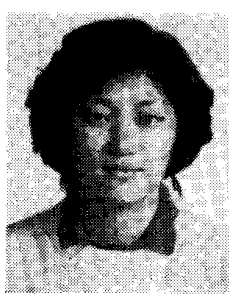

device modeling.
Christina F. Jou was born in Taipei, Taiwan, in 1957. She received the B.S., M.S., and Ph.D degrees in electrical engineering from the University of California, Los Angeles, in 1980, 1982. and 1987, respectively. The subject of her doctoral thesis was the millimeter-wave monolithic Schottky diode-grid frequency doubler.

She is currently employed at Hughes Aircraft Company, Torrance, CA, as a Member of the Technical Staff in the Microwave Products Division, where she is responsible for microwave 
Howard Z. Chen, photograph and biography not available at the time of publication.

Kjell S. Stolt, photograph and biography not available at the time of publication.

\section{事}

Neville C. Luhmann, Jr. received the B S degree in engineering physics from the University of California at Berkeley in 1966 and the Ph.D. degree in physics from the University of Maryland at College Park in 1972.

Following a year at the Princeton Plasma Physics Laboratory, he joined the Electrical Engineering Department at UCLA as an Assistant Professor, subsequently being promoted to Associate Professor in 1978 and Professor in 1981.

Dr. Luhmann is the author of more than 130 scientific papers and approximately 230 scientific presentations in the areas of nearmillimeter-wave imaging; gyrotrons and free-electron lasers; advanced millimeter-wave thermionic sources; near-millimeter-wave $\mathrm{Si}$ and GaAs sources, detectors, and sensors; submillimeter-wave lasers: fusion plasma diagnostics; and nonlinear electromagnetic wave-plasma interactions.

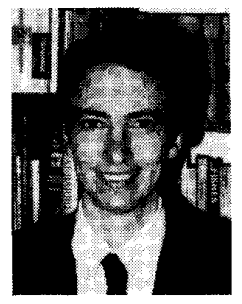

David B. Rutledge (S'75-M'80) was born in Savannah, GA, on January 12,1952 . He received the B.A. degree in mathematics from Williams College in 1973, the M.A. degree in electrical sciences from Cambridge University in 1975, and the Ph.D. degree in electrical engineering from the University of California at Berkeley in 1980.

In 1980 he joined the faculty at the California Institute of Technology, Pasadena, CA, where he is now Associate Professor of Electrical Engineering. He received the IBM faculty development award in 1983 and the NSF Presidential Young Investigator Award in 1984. Dr. Rutledge's research is in developing millimeter- and submillimeter-wave monolithic integrated circuits and applications and in software for computer-aided design and measurement. 